\title{
RESEARCH
}

Open Access

\section{High-throughput screening reveals novel mutations in spinal muscular atrophy patients}

\author{
Ruiping Zhang ${ }^{1 \dagger}$, Chunyu $\mathrm{Gu}^{2 \dagger}$, Linjie Pu ${ }^{2 \dagger}$, Yingtao Meng ${ }^{3}$, Jianbo Shu ${ }^{3,4^{*}}$ and Chunquan $\mathrm{Cai}^{3,4,5^{*}}$
}

\begin{abstract}
Background: Spinal muscular atrophy (SMA) is an autosomal recessive hereditary disease associated with severe muscle atrophy and weakness in the limbs and trunk. The discovery of mutated genes is helpful in diagnosis and treatment for SMA.
\end{abstract}

Methods: Eighty-three whole blood samples were collected from 28 core families of clinically suspected SMA, and multiplex ligation probe amplification (MLPA) was performed. Afterwards, the complete gene sequence of SMN1 gene was detected. Furthermore, 20 SMA patients were selected from the 28 probands, and 5 non SMA children as controls. The Life Technologies SOLiD ${ }^{\mathrm{TM}}$ technology with mate-pair chemistry was utilized to conduct the whole exome high-throughput sequencing.

Results: Twenty-two probands were SMA patients, 3 probands carriers, and 3 probands normal individuals. Moreover, 2 parents from 2 SMA families were with 3 SMN1 exon7 copies. Six SMN1 single nucleotide variants (SNVs) were identified in the 83 samples, and c.[84C > T], c.[271C > T], c.[-39A > G] and g.[70240639G > C] were novel. Compared with control group, 9102 mutation were selected out in SMA patients. SPTA1 mutation c.[-41_40insCTCT], FUT5 SNV c.[1001A > G], and MCCC2 SNV c.[-117A > G] were the 3 most frequent mutations in SMA group (95, 85 and 75\%, respectively).

Conclusions: We identified some mutations in both SMN1 and other genes, and c.[271C > T], c.[-41_-40insCTCT], c. [1001A > G] and c.[-117A > G] might be associated with the onset of SMA.

Keywords: Spinal muscular atrophy (SMA), High-throughput sequencing, SMN1, Onset

\section{Background}

Spinal muscular atrophy (SMA) is an autosomal recessive hereditary disease characterized by degeneration of spinal cord motor neurons, atrophy of skeletal muscles, and generalized weakness [1]. It affects 1 in 10,000 live births, and often leads to early death [2]. SMA manifests over a wide range of severity, affecting infants through adults. According to the onset time and severity of the disease, SMA is

\footnotetext{
*Correspondence: Jianboshu1981@sina.com; caichunquanccq@163.com ${ }^{\dagger}$ Ruiping Zhang, Chunyu Gu and Linjie Pu contributed equally to this work. ${ }^{3}$ Tianjin Pediatric Research Institute, Tianjin Children's Hospital, 238 Longyan Road, Beichen District, Tianjin 300134, China

Full list of author information is available at the end of the article
}

divided into 4 types (SMA1, SMA2, SMA3 and SMA4), and SMA 1, with onset before age 6 months; SMA 2, with onset between age 6 and 18 months; SMA 3, with onset in childhood after age 12 months; and SMA 4, with adult onset [3]. Nusinersen (trade name: Spinraza) is the only approved drug to treat spinal muscular atrophy, which is administered directly to the central nervous system using an intrathecal injection [4], but it's mandatory to have a specific diagnosis in quick time. The genetic profile is essential for the quick and accurate diagnosis of SMA.

SMA is caused by homozygous disruption of the survival motor neuron 1 (SMN1) gene by deletion, conversion, or 
mutation [1]. Since SMA is one of the most common lethal genetic disorders, with a carrier frequency of 1 in 40 to 1 in 60 , direct carrier dosage testing has been beneficial to many families [5]. About 96\% SMA are caused by a homozygous deletion of SMN1 exon7, and the remaining 4\% of cases are caused either by compound heterozygosity with a point mutation in one allele and a deletion in the other or by compound heterozygous point mutations in SMN1 [6]. SMN2 is a homologous gene of SMN1 and functions as a SMA modifier. In general, the copy number of SMN2 is substantial variation in SMA patients, and a high SMN2 copy number tends to a milder type [7]. Furthermore, more and more new genes or novel mutations have been reported to be related to the morbidity, severity, treatment and prognosis of SMA with the development of gene sequencing technology. A study revealed seven different mutations of $S M N 1$, and among them, c.824G > C, and c.825-2A $>\mathrm{T}$ were described for the first time [8]. Another study found the NAIP copy number was inversely correlated with the clinical severity of SMA [9]. GTF2H2 and H4F5 have been proved to be associated with the onset and type of SMA [10]. It is important to understand the genetic characteristics of SMA pathology in order to better understand and diagnose it, so as to implement the nursing care of more and more affected patients.

The severity of SMA varies from infancy to adulthood, which is reflected in the clinical classification system of type 1-4. In addition, a recent study reported that the copy number of SMN genes may determine the pathological type of SMA patients. Cao et al. [11] found that in different copy numbers of $S M N 2$, the distribution of type I and type $2 / 3$ was significantly different $(P<0.001)$ : when the copy number of SMN2 in parents was $1,75 \%$ of SMA progenies were type 1 and $25 \%$ were type $2 / 3$. However, when the parents carried three copies of $S M N 2$, their SMA progenies were all 2/3 type. Therefore, it is very important to understand the pathological genetic characteristics of patients, which directly affects the diagnosis and treatment of patients.

To further improve our understanding of genetic variation in patients with SMA, in this study, multiplex ligation probe amplification (MLPA) was firstly used for preliminary diagnosis in 28 core families of suspected SMA patients, and then the complete gene sequence of SMN1 gene was detected by high-throughput sequencing to find more mutations in the 28 core families. Afterwards, 20 children diagnosed with SMA and 5 children diagnosed with non SMA were enrolled, and the whole exome screening of other related genes was performed to explore more genes and mutations involved in the onset of SMA.

\section{Methods}

\section{Patients and samples}

From December 2013 to May 2017, 28 probands of clinically suspected SMA, 15 males and 13 females, were accepted by our hospital (Tianjin Children's Hospital, Tianjin, China) because of unstable walking, and their age ranging from 1 month to 12 years. The phenotypes of their parents were normal. $3-5 \mathrm{ml}$ peripheral blood samples from the 83 enrolled cases (probands and their parents) were collected. All the subjects signed the informed consent forms for genetic testing routinely, and all procedures were in accordance with the ethical standards of the institutional and/or national research committee.

\section{MLPA}

Extracted Genomic DNA with the salting out method. The nucleic acid quantitative instrument NADO DROP 2000 (Thermo Fisher Scientific Inc., Waltham, USA) was utilized to determine the quality and quantity of the extracted DNA. MLPA was performed using a SALSA MLPA Kit P021 (MRC-Holland, Amsterdam, Netherlands) according to the manufacturer's protocol. MLPA products were run on an ABI PRISM 3130 genetic analyzer (Applied Biosystems International Inc., California, USA). The evaluation criteria were based on the kit instructions: in normal individuals, SMN1 exon7 is 2 copies; the SMN1 gene of patients with homozygous deletion and carriers with heterozygous deletion is 0 copies and 1copy, respectively $[12,13]$.

\section{SMN1 gene screening by high-throughput sequencing}

The complete genome sequence of SMN1 gene was detected from above 83 enrolled cases via high-throughput sequencing. Firstly, high-molecular-weight genomic DNAs were extracted with the DNeasy Blood and Tissue kit (QIAGEN, Dusseldorf, Germany) and $10 \mu \mathrm{g}$ genomic DNA was used for library generation according to the manufacturer's recommendations. Secondly, the Illumina HiSeq-2500 platform (Illumina, California, USA) was used to conduct the sequencing reaction based on the manufacturer's protocol. The average sequencing depth was $100 \times$, and more than $96 \%$ regions was up to $20 \times$. Thirdly, the raw data were compared with human genome (NCBI build 36, hg18), and marked repeated reads and filtered out low-quality data. Fourthly, the base quality of reads was calibrated again by the genome analysis toolkit (GATK) algorithm, and ultimately all the a single base of DNA (point mutation) or a loss of base pairs (deletion) were screened out with the GATK software V3.0 (Eli and Edythe L. Broad Institute, Massachusetts, USA) [14]. The $\mathrm{QC}_{30}$ of the raw data was more than $85 \%$ in all samples, and the allele frequency of all mutations was more than $20 \%$.

\section{The whole exome screening by high-throughput sequencing}

Based on previous results, 20 SMA patients with homozygous deletion of SMN1 exon7 were selected from the 28 probands, and 5 non SMA children were as controls ( 2 children carried with heterozygous deletion of SMN1 
exon7 and 3 children with 2 SMN1 exon7 copies). Whole exome high-throughput sequencing was performed by The Life Technologies $\mathrm{SOLiD}^{\mathrm{mm}}$ (version 3) technology with mate-pair chemistry. $20 \mu \mathrm{g}$ highmolecular-weight genomic DNAs was used for library generation. The raw data were analyzed with GATK software. The average sequencing depth was $100 \times$, and more than $96 \%$ regions was up to $20 \times$. The $\mathrm{QC}_{30}$ of the raw data was more than $85 \%$ in all samples, and the allele frequency of all mutations was more than $40 \%$.

\section{Results}

\section{MLPA}

MLPA results showed that 22 probands were with homozygous deletion of SMN1 exon7 (SMA patients), 3 probands carried with heterozygous deletion of SMN1 exon7 (carriers), and 3 probands with 2 SMN1 copies (normal individuals). However, the mother of a SMA patient had 3 SMN1 exon7 copies and the father was carrier, and the father of another SMA patient had 3 SMN1 exon7 copies and the mother was carrier. Here, we recorded the above 2 SMA patients as Proband-1 and Proband-2, respectively. Furthermore, 1 carrier, a 2-year old girl, had some clinical features that correspond to SMA, such as atrophy of skeletal muscles, generalized weakness and extensive neurogenic injury by electromyography. Here, the carrier was named as Proband-3. Besides, the father of Proband-3 was without heterozygous deletion of SMN1 exon7 and exon8, and the mother was a carrier.

\section{Novel mutations of SMN1 in suspected SMA family}

A total of 6 single nucleotide variants (SNVs) of SMN1 were identified in the 83 samples, and they were showed in Table 1. Three SNVs located in exon, 1 in UTR5 and 2 in intron; c. $[84 \mathrm{C}>\mathrm{T}]$, c. $[271 \mathrm{C}>\mathrm{T}]$, c. $[-39 \mathrm{~A}>\mathrm{G}]$ and g. $[70240639 \mathrm{G}>\mathrm{C}]$ were firstly reported here; c. [84C $>\mathrm{T}]$ and c. $[462 \mathrm{~A}>\mathrm{G}]$ were synonymous mutations, and c. $[271 \mathrm{C}>\mathrm{T}]$ were stop-gain; c. [271C $>\mathrm{T}]$ caused changes in encoded amino acids. Furthermore, c. [271C $>\mathrm{T}]$ was found in Proband-3 and her father, and c. $[462 \mathrm{~A}>\mathrm{G}]$ was occurred in 17 SMA patients, 2 carrier and 2 normal individuals. In addition, 1 carrier was with c. $[84 \mathrm{C}>\mathrm{T}], 1$
SMA patient with c.[-39A > G], and 1 SMA patient with g.[70240639G > C].

\section{Novel mutations occurred in only SMA patients}

Compared with control group, a total of 9102 mutation were selected out in SMA patients with homozygous deletion of SMN1 exon7. They were located in the exon region, and occurred only in SMA patients not in carriers and normal individuals. Among them, 2415 genes and some indefinite genes were included, and 8619 SNVs, 267 deletions and 216 inserts were contained. Here, the indefinite genes were removed, and the 30 most frequent mutations were showed in Table 2 (Frequency $\geq 50 \%$ ). It was obvious from Table 2 that only the $M C C C 2$ missense mutation c. [1001A $>$ G] located on chromosome $5 \mathrm{q} 13$, which was the same location as SMN1 and SMN2. The others were unlinked to $5 \mathrm{q} 13$.

\section{Discussion}

Since both the clinical features and the mutation characteristics of SMA are obvious, the diagnosis of SMA is considered as relatively easy process. However, a recent review reported that the diagnostic process for SMA is not always simple, and there is usually a delay between the onset of clinical features and the diagnosis in all types of SMA [15]. At present, MLPA is the gold standard for clinical diagnosis of SMA. However, MLPA can only detect the deletion of SMN1 according to the gene copy number, not detect point mutations of SMN1. It is well known that about $4 \%$ of SMA patients bear one SMN1 copy with an intragenic mutation. Therefore, some SMA patients are inevitably misdiagnosed as carriers. In addition, the emergence of new therapies increases the need for early diagnosis of SMA, the approval of therapies and the neonatal screening programs urgently require a more detailed understanding of genetic variation [16]. In this study, Proband-3 was with one SMN1 copy and the SMN1 stop-gain mutation c. $[271 \mathrm{C}>\mathrm{T}]$, and the heterozygous deletion of SMN1 exon 7 was from her mother, and the SMN1 stop-gain mutation c. [271C > T] from her father. The SMN1 stopgain mutation c.[271C $>\mathrm{T}$ ] was never reported before, and it led to a amino acid change. Although MLPA

Table 1 Mutations in SMN1

\begin{tabular}{|c|c|c|c|c|c|c|c|c|}
\hline Position & MT & Ex-In & Function & REF $>$ ALT & dbSNP & cHGVS & AAChange & Frequency \\
\hline $70,234,668$ & snv & exon2 & synonymous & $C>T$ & NA & c. $[84 C>T]$ & NA & $3.61 \%(3 / 83)$ \\
\hline $70,220,892$ & snv & exon3 & stop-gain & $C>T$ & NA & c. $[271 \mathrm{C}>\mathrm{T}]$ & p.[Gln91X] & $2.41 \%(2 / 83)$ \\
\hline $70,238,373$ & snv & exon4 & synonymous & $A>G$ & rs4915 & c. $[462 A>G]$ & NA & $83.13 \%(69 / 83)$ \\
\hline $70,220,892$ & snv & UTR5 & unknown & $A>G$ & NA & c. $[-39 A>G]$ & NA & $4.82 \%(4 / 83)$ \\
\hline $70,240,639$ & snv & intron & unknown & $G>C$ & NA & g.[70240639G > C] & NA & $8.43 \%(7 / 83)$ \\
\hline $70,247,937$ & snv & intron & unknown & $A>C$ & rs200563560 & g.[70247937A > C] & NA & $1.20 \%(1 / 83)$ \\
\hline
\end{tabular}

MT Mutation type, Ex-In Exon or intron, snv Single nucleotide variant, NA No report or no change, $X$ Unknown amino acids, Frequency Frequency in all samples 
Table 2 The 30 most frequent mutations in SMA patients

\begin{tabular}{|c|c|c|c|c|c|c|c|c|c|}
\hline Gene & Position & MapLoc & MT & ExID & Function & cHGVS & AAChange & dbSNP & Frequency \\
\hline SPTA1 & $158,656,347$ & $1 \mathrm{q} 23.1$ & ins & EX1 & utr-5 & c.[-41_-40insCTCT] & NA & rs111674514 & $95 \%(19 / 20)$ \\
\hline FUT5 & $5,866,736$ & $19 p 13.3$ & snv & EX2E & missense & c. $[1001 \mathrm{~A}>\mathrm{G}]$ & p.[His334Arg] & rs778984 & $85 \%(17 / 20)$ \\
\hline MCCC2 & $70,883,136$ & $5 q 13.2$ & snv & EX1 & utr-5 & c. $[-117 \mathrm{~A}>\mathrm{G}]$ & NA & rs11746722 & $75 \%(15 / 20)$ \\
\hline ARL14EP & $30,352,473$ & $11 p 14.1$ & ins & EX2 & utr-5 & c. $\left[-23 \_-22\right.$ ins AG $]$ & NA & rs75725983 & $70 \%(14 / 20)$ \\
\hline GNS & $65,146,532$ & $12 q 14.3$ & snv & EX2 & coding-synon & c. $[198 \mathrm{G}>\mathrm{A}]$ & NA & rs1147096 & $65 \%(13 / 20)$ \\
\hline COL3A1 & $189,864,080$ & $2 q 32.2$ & snv & EX30 & missense & c. $[2092 \mathrm{G}>\mathrm{A}]$ & p.[Ala698Thr] & rs1800255 & $65 \%(13 / 20)$ \\
\hline HSPD1 & $198,363,504$ & $2 q 33.1$ & snv & EX2 & coding-synon & c. $[69 \mathrm{~T}>\mathrm{C}]$ & NA & rs1050347 & $65 \%(13 / 20)$ \\
\hline SREBF1 & $17,714,719$ & $17 p 11.2$ & del & EX20E & utr-3 & c.[1217_1218delTT] & NA & NA & $60 \%(12 / 20)$ \\
\hline CYP2A6 & $41,355,849$ & $19 q 13.2$ & snv & EX2 & coding-synon & c. $[217 \mathrm{~T}>\mathrm{C}]$ & NA & rs2302990 & $60 \%(12 / 20)$ \\
\hline SDHAP1 & $195,710,975$ & $3 q 29$ & snv & IVS5 & unknown & n. $[750+8 C>T]$ & NA & NA & $60 \%(12 / 20)$ \\
\hline LHX4 & $180,199,727$ & $1 \mathrm{q} 25.2$ & snv & EX1 & coding-synon & c. $[63 \mathrm{~T}>\mathrm{C}]$ & NA & rs75857235 & $55 \%(11 / 20)$ \\
\hline RGPD8 & $113,147,159$ & $2 q 13$ & snv & EX20 & coding-synon & c. $[3363 C>T]$ & NA & rs62157473 & $55 \%(11 / 20)$ \\
\hline PROM1 & $16,020,162$ & $4 p 15.32$ & snv & EX8 & coding-synon & c. $[786 \mathrm{G}>\mathrm{A}]$ & NA & rs2286455 & $55 \%(11 / 20)$ \\
\hline HLA-DQB2 & $32,726,838$ & 6p21.32 & snv & EX3 & coding-synon & c. $[435 G>A]$ & NA & rs17850863 & $55 \%(11 / 20)$ \\
\hline ERCC6 & $50,666,808$ & $10 q 11.23$ & snv & EX21E & utr-3 & c. $\left[{ }^{*} 53 \mathrm{~T}>\mathrm{C}\right]$ & NA & rs4253231 & $50 \%(10 / 20)$ \\
\hline $\mathrm{NOTCH} 2$ & $120,612,004$ & $1 \mathrm{p} 11.2$ & del & EX1 & frameshift & c.[17_18delCC] & p.[Pro6Arg] & NA & $50 \%(10 / 20)$ \\
\hline GORAB & $170,521,650$ & $1 \mathrm{q} 24.2$ & snv & EX5E & utr-3 & C. $\left[{ }^{*} 47 \mathrm{~T}>\mathrm{G}\right]$ & NA & rs7552922 & $50 \%(10 / 20)$ \\
\hline DYNC1H1 & $102,493,761$ & $14 q 32.31$ & snv & EX46 & coding-synon & c. $[8928 \mathrm{~A}>\mathrm{G}]$ & NA & rs8010870 & $50 \%(10 / 20)$ \\
\hline ACSF3 & $89,167,138$ & $16 q 24.3$ & snv & EX3 & missense & c. $[49 \mathrm{G}>\mathrm{C}]$ & p.[Ala17Pro] & rs11547019 & $50 \%(10 / 20)$ \\
\hline MYO5B & $47,429,022$ & $18 q 21.1$ & snv & EX21 & missense & c. $[2753 \mathrm{G}>\mathrm{A}]$ & p.[Arg918His] & rs2298624 & $50 \%(10 / 20)$ \\
\hline MYO5B & $47,500,836$ & $18 q 21.1$ & snv & EX10 & coding-synon & c. $[1206 C>T]$ & NA & rs11082795 & $50 \%(10 / 20)$ \\
\hline BCL10 & $85,742,157$ & $1 \mathrm{p} 22.3$ & snv & EX1 & utr-5 & c. $[-122 C>\mathrm{G}]$ & NA & rs1060843 & $50 \%(10 / 20)$ \\
\hline CGB5 & $49,547,446$ & $19 q 13.33$ & snv & EX1 & utr-5 & c. $[-21 \mathrm{G}>\mathrm{C}]$ & NA & rs35014217 & $50 \%(10 / 20)$ \\
\hline SNRNP200 & $96,952,833$ & $2 q 11.2$ & snv & EX27 & coding-synon & c.[3550 T > C] & NA & rs3171927 & $50 \%(10 / 20)$ \\
\hline NR3C2 & $149,002,017$ & $4 q 31.23$ & del & EX9E & utr-3 & c.[478delA]] & NA & rs10708334 & $50 \%(10 / 20)$ \\
\hline GRM1 & $146,755,560$ & $6 q 24.3$ & snv & EX9E & coding-synon & c.[3213 T > G] & NA & rs1047006 & $50 \%(10 / 20)$ \\
\hline RP1L1 & $10,467,576$ & $8 p 23.1$ & snv & EX4E & coding-synon & c. $[4032 \mathrm{~A}>\mathrm{G}]$ & NA & rs4840499 & $50 \%(10 / 20)$ \\
\hline RP1L1 & $10,467,652$ & $8 p 23.1$ & snv & EX4E & missense & c. $[3956 C>$ G] & p.[Ala1319Gly] & rs4840501 & $50 \%(10 / 20)$ \\
\hline CELP & $135,961,787$ & $9 q 34.2$ & snv & EX4 & ncRNA & n. $[622 A>G]$ & NA & rs10901232 & $50 \%(10 / 20)$ \\
\hline CELP & $135,961,796$ & $9 q 34.2$ & snv & EX4 & ncRNA & n. $[631 C>T]$ & NA & rs10901233 & $50 \%(10 / 20)$ \\
\hline
\end{tabular}

MapLoc The locations on chromosome, MT Mutation type, Ex Exon, snv Single nucleotide variant, ins Insert, del deletion, NA no report or no change, Frequency Frequency in the 20 SMA samples

results showed Proband-3 to be carrier, some SMArelated clinical features were occurred on her. Here, we suspected that Proband-3 might be a SMA patient caused by the heterozygous deletion of SMN1 exon 7 combined with the SMN1 stop-gain mutation c.[271C > T]. Simultaneously, c. $[271 \mathrm{C}>\mathrm{T}]$ might be involved in the onset of SMA. In addition, $[2+0]$ genotype carriers are two SMN1 copies on one chromosome and with deletion of SMN1 on the other chromosome [17]. In this article, we found 2 patients (Proband- 1 and Proband-2) whose one parent was carriers and the other parent with 3 SMN1 exon7 copies (Proband-1's mother and Proband-2's father). Based on our results we suspected that Proband-1's mother and Proband-2's father might be $[2+0]$ genotype carriers.

SMN1 and SMN2 present on chromosome 5q13, and of the 5q13-linked SMA patients, $96.4 \%$ show homozygous absence of SMN1 exons 7 and 8 or exon 7 only, whereas $3.6 \%$ present a compound heterozygosity with a subtle mutation on one chromosome and a deletion/gene conversion on the other chromosome [6]. Due to the complexity of $5 \mathrm{q}$ chromosome structure, the mechanism of SMA has not been fully elucidated. For example, the rare variations in SMN2 have been described by several studies [18-20], and some scholars believe that the variations in SMN2 locus, such as the deletion of adjacent 
NAIP1 gene, will affect or even change the severity of SMA [21, 22]. With the development of bioinformatics, more and more mutations have been discovered in SMN1, and some of them possess significant clinical implications. Yamamoto et al. [23] revealed 4 intragenic mutations (p.Ala2Val, p.Trp92Ser, p.Thr274TyrfsX32 and p.Tyr27 7Cys), and location of the mutations were associated with the clinical severity of SMA. Ronchi et al. [24] described a novel SMN1 mutation that affected the donor splice site of exon 7 and resulted in an unusually severe SMA phenotype with rapid fatal outcome in an Italian infant. In this article, we found 6 SMN1 SNVs in 28 core families of suspected SMA patients, including 4 novel mutations c. $[84 \mathrm{C}>\mathrm{T}]$, c. $[271 \mathrm{C}>\mathrm{T}], \quad$ c. $[-39 \mathrm{~A}>\mathrm{G}]$ and g. $[7024063$ $9 \mathrm{G}>\mathrm{C}]$, which had never been previously reported. Besides, we identified more mutations combined with homozygous absence of SMN1 exons7 (Table 2). The 3 most frequent mutations were the insertion mutation c.[-41_40insCTCT] in SPTA1 exon1 (rs111674514), the SNV c. [1001A > G] in FUT5 exon2 (rs778984), and the SNV c. $[-117 \mathrm{~A}>\mathrm{G}]$ in $M C C C 2$ exon1 (rs11746722). SPTA1 encodes the human erythroid alpha-spectrin, which is an actin crosslinking and molecular scaffold protein that links the plasma membrane to the actin cytoskeleton, and functions in the determination of cell shape, arrangement of transmembrane proteins, and organization of organelles $[25,26]$. Mutations in SPTA1 can lead to a variety of hereditary red blood cell disorders, including elliptocytosis type 2, pyropoikilocytosis, and spherocytic hemolytic anemia [27, 28]. FUT5 encodes alpha1,3-fucosyltransferase in human, the down-regulation of FUT5 reduces the expression of sialyl-Lewis antigens and the adhesion and binding capacities of gastric cancer cells [29]. Gene transfer of alpha1,3-fucosyltransferase increased tumor growth of the PC-3 human prostate cancer cell line through enhanced adhesion to prostatic stromal cells [30]. Methylcrotonyl CoA carboxylase $\beta$ (MCC $\beta$ ) is encoded by $M C C C 2$, and point mutations and deletion events in $M C C 2$ can lead to MCC deficiency [31]. MCC deficiency is a rare autosomal recessive genetic disorder whose clinical presentations range from benign to profound metabolic acidosis and death in infancy, which is has something in common with SMA in some ways. MCCC2 locates on chromosome $5 \mathrm{q} 13$, which was the same as SMN1. Some studies indicated that SMN1 was the causative gene, and other genes on $5 \mathrm{q} 13$ region acted as modifier gene (such as SMN2, NAIP and GTF2H2), which were associated with disease severity [32]. The mutations rs111674514 in SPTA1, rs778984 in FUT5 and rs1174 6722 in $M C C C 2$ have been identified previously, but the clinical significance remains uncertain. In this article, we found they were widely prevalent in SMA patients, and almost nonexistent in non-patients. Therefore, it suggested they might be involved in the morbidity of SMA.
One limitation of this study is the failure to use healthy children as control. The reason is that the children involving in this study are younger ( 0 to 12 years old), many parents are reluctant to let their healthy children participate in the study because of insufficient understanding of genetic testing. Another objective reason is that the cost of sequencing is high, so parents of healthy children are unwilling to pay for it. However, in terms of the rationality of the entire study, we really should add healthy children as control to make our research more complete, and that is what we will improve in the follow-up related studies. In contrast, using nonSMA patients with similar clinical characteristics of SMA as a control could exclude some genetic mutations that may lead to similar clinical characteristics of children with SMA, so as to make the object of this study more accurate. Using this population as a control can exclude other mutations that may cause dyskinesia, and only retain mutations unique to SMA patients.

\section{Conclusions}

We found more mutations in both SMN1 and other genes, and some of them were associated with the onset of SMA, such as the SMN1 stop-gain mutation c. $[271 \mathrm{C}>\mathrm{T}]$, the SPTA1 insertion mutation c.[-41_40insCTCT], the FUT5 SNV c. $[1001 \mathrm{~A}>\mathrm{G}]$, and the MCCC2 SNV c. [-117A > G].

\section{Abbreviations \\ SMA: Spinal muscular atrophy; MLPA: Multiplex ligation probe amplification; SNVs: Single nucleotide variants; SMN: Survival of motor neuron; NAIP: Neuronal apoptosis inhibitory protein; GATK: Genome analysis toolkit; MCC $\beta$ : Methylcrotonyl CoA carboxylase $\beta$}

\section{Acknowledgments}

We would like to thank all the participants and their families who took part in this research.

\section{Authors' contributions}

CQC and JBS designed the study and drafted the initial manuscript, and revised the manuscript. RPZ participated in the design of study and critically reviewed the manuscript. CYG and LIP collected samples and critically reviewed the manuscript. CYG, LJP and YTM analyzed and interpreted data and reviewed the manuscript. All authors provided substantial contributions to the conception or design of the work, or the acquisition, analysis, or interpretation of data for the paper, revised the manuscript for important intellectual content, approved the final version, and agreed to be accountable for all aspects of the work.

\section{Funding}

This work was supported by the National Natural Science Foundation of China [grant number 81771589]; the Program of Tianjin Science and Technology Plan [grant number 18ZXDBSY00170]; and the Key Project of Tianjin Health Care Professionals [grant number 16KG166]. The fund body was not involved in the project design, data collection, analysis, etc.

\section{Availability of data and materials}

The datasets used and/or analyzed during the current study are available from the corresponding author on reasonable request.

Ethics approval and consent to participate

All the subjects signed the informed consent forms for genetic testing routinely, and the study was approved by the medical ethics committee of 
Tianjin Children's Hospital. A written informed consent was obtained from the parents and informed assent from the children and adolescents.

\section{Consent for publication}

Not applicable.

\section{Competing interests}

The authors declare that they have no competing interests.

\section{Author details}

${ }^{1}$ Department of Pediatric, Tianjin Children's Hospital, Tianjin 300134, China. ${ }^{2}$ Graduate School, Tianjin Medical University, Tianjin 300070, China. ${ }^{3}$ Tianjin Pediatric Research Institute, Tianjin Children's Hospital, 238 Longyan Road, Beichen District, Tianjin 300134, China. ${ }^{4}$ Tianjin Key Laboratory of Birth Defects for Prevention and Treatment, Tianjin 300134, China. ${ }^{5}$ Department of Neurosurgery, Tianjin Children's Hospital, 238 Longyan Road, Beichen District, Tianjin 300134, China.

Received: 6 April 2020 Accepted: 26 October 2020

Published online: 04 November 2020

\section{References}

1. Lunn MR, Wang CH. Spinal muscular atrophy. Lancet. 2008;371:2120-33.

2. Sheng-Yuan Z, Xiong F, Chen YJ, Yan TZ, Zeng J, Li L, et al. Molecular characterization of SMN copy number derived from carrier screening and from core families with SMA in a Chinese population. Eur J Hum Genet. 2010;18:978-84

3. Kolb SJ, Kissel JT. Spinal muscular atrophy. Neurol Clin. 2015;33:831-46.

4. Ottesen EW. ISS-N1 makes the first FDA-approved drug for spinal muscular atrophy. Transl Neurosci. 2017;8:1-6.

5. Prior TW, Nagan N. Spinal muscular atrophy: overview of molecular diagnostic approaches. Curr Protoc Hum Genet. 2016;88:9-27.

6. Wirth B. An update of the mutation spectrum of the survival motor neuron gene (SMN1) in autosomal recessive spinal muscular atrophy (SMA). Hum Mutat. 2000;15:228-37.

7. Stabley DL, Harris AW, Holbrook J, Chubbs NJ, Lozo KW, Crawford TO, et al. SMN1 and SMN2 copy numbers in cell lines derived from patients with spinal muscular atrophy as measured by array digital PCR. Mol Genet Genomic Med. 2015;3:248-57.

8. Zabnenkova W, Dadali EL, Artemieva SB, Sharkova IV, Rudenskaya GE, Polyakov AV. SMN1 gene point mutations in type I-IV proximal spinal muscular atrophy patients with a single copy of SMN1. Genetika. 2015;51: 1075-82.

9. He J, Zhang QJ, Lin QF, Chen YF, Lin XZ, Lin MT, et al. Molecular analysis of SMN1, SMN2, NAIP, GTF2H2, and H4F5 genes in 157 Chinese patients with spinal muscular atrophy. Gene. 2013;518:325-9.

10. Zeng G, Zheng H, Cheng J, Chen R, Lin H, Yang J, et al. Analysis and carrier screening for copy numbers of SMN and NAIP genes in children with spinal muscular atrophy. Chinese J Med Genet. 2014;31:152-5.

11. Cao Y, Qu Y, Bai J, Cheng M, Jin Y, Wang H, et al. Transmission characteristics of SMN from 227 spinal muscular atrophy core families in China. J Hum Genet. 2020:5:1-5.

12. Liu Z, Zhang P, He X, Liu S, Tang S, Zhang R, et al. New multiplex real-time PCR approach to detect gene mutations for spinal muscular atrophy. BMC Neurol. 2016;16:141.

13. Sheth J, Mistri M, Bhavsar R, Pancholi D, Kamate M, Gupta N, et al. Batten disease: biochemical and molecular characterization revealing novel PPT1 and TPP1 gene mutations in Indian patients. BMC Neurol. 2018;18:203 Chen $X$, Tong $Y$, Shi Z, Chen H, Yang Z, Wang Y, et al. Noninvasive molecular diagnosis of craniopharvngioma with MRI-based radiomics approach. BMC Neurol. 2019:19:6.

14. Chen $X$, Tong $Y$, Shi $Z$, Chen $H$, Yang $Z$, Wang $Y$, et al. Noninvasive molecular diagnosis of craniopharvngioma with MRI-based radiomics approach. BMC Neurol. 2019;19:6.

15. Lin CW, Kalb SJ, Yeh WS. Delay in diagnosis of spinal muscular atrophy: a systematic literature review. Pediatr Neurol. 2015;4:293-300.

16. Pera MC, Coratti G, Berti B, D'Amico A, Sframeli M, Albamonte E, et al. Diagnostic journey in spinal muscular atrophy: is it still an odyssey? PLoS One. 2020;3:e0230677.
17. Ar Rochmah M, Awano H, Awaya T, Harahap NIF, Morisada N, Bouike Y, et al. Spinal muscular atrophy carriers with two SMN1 copies. Brain and Development. 2017;39:851-60.

18. Wu X, Wang SH, Sun J, Krainer AR, Hua Y, Prior TW. A-44G transition in SMN2 intron 6 protects patients with spinal muscular atrophy. Hum Mol Genet. 2017;26:2768-80.

19. Calucho M, Bernal S, Alias L, March F, Vencesla A, Rodriguez-Alvarez FJ, et al. Correlation between SMA type and SMN2 copy number revisited: an analysis of 625 unrelated Spanish patients and a compilation of 2834 reported cases. Neuromuscul Disord. 2018;28:208-15.

20. Ruhno C, McGovern VL, Avenarius MR, Snyder PJ, Prior TW, Nery FC, et al. Complete sequencing of the SMN2 gene in SMA patients detects SMN gene deletion junctions and variants in SMN2 that modify the SMA phenotype. Hum Genet. 2019;138:241-56.

21. Riessland M, Kaczmarek A, Schneider S, Swoboda KJ, Lohr H, Bradler C, et al. Neurocalcin delta suppression protects against spinal muscular atrophy in humans and across species by restoring impaired endocytosis. Am J Hum Genet. 2017;100:297-315.

22. Wadman RI, Jansen MD, Curial CAD, Groen EJN, Stam M, Wijngaarde CA, et al. Analysis of FUS, PFN2, TDP-43, and PLS3 as potential disease severity modifiers in spinal muscular atrophy. Neurol Genet. 2020;6:e386.

23. Yamamoto T, Sato H, Lai PS, Nurputra DK, Harahap NI, Morikawa S, et al. Intragenic mutations in SMN1 may contribute more significantly to clinical severity than SMN2 copy numbers in some spinal muscular atrophy (SMA) patients. Brain and Development. 2014;36:914-20.

24. Ronchi D, Previtali SC, Sora MG, Barera G, Del Menico B, Corti S, et al. Novel splice-site mutation in SMN1 associated with a very severe SMA-I phenotype. J Mol Neurosci. 2015;56:212-5.

25. Govindaraj $\vee$, Rao AJ. Proteomic identification of non-erythrocytic alphaspectrin-1 down-regulation in the pre-optic area of neonatally estradiol-17beta treated female adult rats. Horm Mol Biol Clin Investig. 2016;26:165-72.

26. Speicher DW. Structural and functional features of the alpha-1 domain from human erythrocyte spectrin. Prog Clin Biol Res. 1984;165:441-56.

27. Niss O, Chonat S, Dagaonkar N, Almansoori MO, Kerr K, Rogers ZR, et al. Genotype-phenotype correlations in hereditary elliptocytosis and hereditary pyropoikilocytosis. Blood Cells Mol Dis. 2016;61:4-9.

28. Han E, Kim A, Park J, Kim M, Kim Y, Han K, et al. Spectrin Tunis (Sp alpha (I/ 78)) in a Korean family with hereditary elliptocytosis. Ann Lab Med. 2013;33: 386-9.

29. Padro M, Cobler L, Garrido M, de Bolos C. Down-regulation of FUT3 and FUT5 by shRNA alters Lewis antigens expression and reduces the adhesion capacities of gastric cancer cells. Biochim Biophys Acta. 1810;2011:1141-9.

30. Inaba Y, Ohyama C, Kato T, Satoh M, Saito H, Hagisawa S, et al. Gene transfer of alpha1,3-fucosyltransferase increases tumor growth of the PC-3 human prostate cancer cell line through enhanced adhesion to prostatic stromal cells. Int J Cancer. 2003;107:949-57.

31. Morscher RJ, Grunert SC, Burer C, Burda P, Suormala T, Fowler B, et al. A single mutation in MCCC1 or MCCC2 as a potential cause of positive screening for 3-methylcrotonyl-CoA carboxylase deficiency. Mol Genet Metab. 2012;105:602-6.

32. Theodorou L, Nicolaou P, Koutsou P, Georghiou A, Anastasiadou V, Tanteles $G$, et al. Genetic findings of Cypriot spinal muscular atrophy patients. Neurol Sci. 2015;36:1829-34.

\section{Publisher's Note}

Springer Nature remains neutral with regard to jurisdictional claims in published maps and institutional affiliations.

\section{Ready to submit your research? Choose BMC and benefit from:}

- fast, convenient online submission

- thorough peer review by experienced researchers in your field

- rapid publication on acceptance

- support for research data, including large and complex data types

- gold Open Access which fosters wider collaboration and increased citations

- maximum visibility for your research: over $100 \mathrm{M}$ website views per year

At BMC, research is always in progress.

Learn more biomedcentral.com/submission 